\title{
VGI in National Mapping Agencies: Experiences and Recommendations
}

\author{
Ana-Maria Olteanu-Raimond ${ }^{\star}$, Mari Laakso ${ }^{\dagger}$, \\ Vyron Antoniou ${ }^{\ddagger}$, Cidália Costa Fonte ${ }^{\S}$, \\ Alexandra Fonseca', Magdalena Grus", \\ Jenny Harding ${ }^{\star \star}$, Tobias Kellenberger ${ }^{\dagger \dagger}$, \\ Marco Minghini ${ }^{\ddagger \ddagger}$, Andriani Skopeliti ${ }^{\S \S}$
}

*Univ. Paris-Est, LASTIG COGIT, IGN, ENSG, F-94160 Saint-Mande, France, ana-maria.raimond@ign.fr

${ }^{\dagger} F i n n i s h$ Geospatial Research Institute, Kirkkonummi 02430, Finland ${ }^{*}$ Hellenic Army General Staff, Geographic Directorate, PAPAGOU Camp, Mesogeion 227-231, Cholargos, 15561, Greece

${ }^{s}$ Department of Mathematics, University of Coimbra, 3001-501 Coimbra, Portugal / INESC Coimbra, Rua Sílvio Lima, Pólo II, 3030-290 Coimbra, Portugal

'Portuguese Territorial Development Agency (DGT), Center for Environmental and Sustainability Research (CENSE), Portugal

"Kadaster, Hofstraat 110, 7311 KZ Apeldoorn, the Netherlands

** Ordnance Survey, Adanac Drive, Southampton SO16 0AS, UK

${ }^{\dagger \dagger}$ Federal Office of Topography swisstopo, Seftigenstrasse 264,

3084 Wabern, Switzerland

${ }^{\ddagger}$ Department of Civil and Environmental Engineering, Politecnico di Milano,

Piazza Leonardo da Vinci 32, 20133 Milano, Italy

${ }^{s s}$ School of Rural and Surveying Engineering, National Technical

University of Athens, 9 H. Polytechniou, Zografou, 15780, Greece

How to cite this book chapter:

Olteanu-Raimond, A-M, Laakso, M, Antoniou, V, Fonte, C C, Fonseca, A, Grus, M, Harding, J, Kellenberger, T, Minghini, M, Skopeliti, A. 2017. VGI in National Mapping Agencies: Experiences and Recommendations. In: Foody, G, See, L, Fritz, S, Mooney, P, Olteanu-Raimond, A-M, Fonte, C C and Antoniou, V. (eds.) Mapping and the Citizen Sensor. Pp. 299-326. London: Ubiquity Press. DOI: https://doi.org/10.5334/bbf.m. License: CC-BY 4.0 


\begin{abstract}
Despite the considerable growth in Volunteered Geographic Information (VGI) activities in citizen sensing and the evident opportunities for VGI use in map revision and updating, few European National Mapping Agencies (NMAs) or other types of government bodies have engaged significantly with VGI. Moreover, the level of engagement of NMAs with the VGI community varies greatly, and most of them have proposed their own tools for encouraging citizens and public partners to collect feedback or new data. There are numerous barriers limiting the participation of citizens and public partners in NMA data collection, including data quality issues, the motivation of the contributors and legal issues. The aim of this chapter is to give an overview of the experiences of some European NMAs in engaging with VGI. Guidelines and recommendations to support wider engagement with the VGI community are also proposed to help NMAs and interested government bodies exploit the potential of VGI for authoritative mapping.
\end{abstract}

\title{
Keywords
}

VGI, authoritative mapping, VGI platform, data collection, data quality

\section{Introduction}

Volunteered Geographic Information (VGI) initiatives have seen considerable growth in citizen sensing (Goodchild, 2007). Different terms are used in the literature to describe this volunteered activity, such as crowdsourcing and neogeography (Turner, 2006) or user generated spatial content (Antoniou et al., 2009). See et al. (2016) give a complete review on the current terminologies used and the distinctions between them. In this chapter, the focus is on VGI in the context of European National Mapping Agencies (NMAs).

With the adoption of the Open Data Policy ${ }^{1}$ which encourages to freely release data that can be used and republished by any user, many government datasets are now freely available to the public, including spatial data from some European NMAs (Brovelli et al., 2016). Some NMAs, such as those of Finland and the Netherlands, have released their datasets under open access licences; these authoritative data have been integrated into OpenStreetMap (OSM), which has improved the OSM database. More studies are necessary to determine if this integration may also have benefits for NMAs. The Open Data Policy can be an opportunity for both NMAs and geographic data end users. Indeed, releasing data under open access licences through a platform can increase the usability of authoritative data, because end users such as citizens can freely download and use data for different purposes. In addition, the 
motivation for citizens and partners to contribute by adding new information, giving feedback and providing alerts on errors and updates can also increase.

Although local governments had already started during the last ten years to use VGI as a participation platform to engage in a dialogue with citizens rather than as a way to simply gain or share information (Johnson and Sieber, 2013), there has been a noticeable change. Indeed, more recently, different initiatives have been proposed by local governments to collect data for different purposes (such as in urban planning, in order to advertise new regulations) where citizens have been considered both as sensors and as potential partners (Karimipour and Azari, 2015; Sedano, 2016).

Traditionally, almost all mapping agencies have some experience in collecting information from their data users by receiving alerts regarding mapping errors or updates. However, it is important to differentiate between passive processes and more active processes in which the mapping agencies actively engage with the VGI community by proposing platforms to collect and disseminate data (See et al., 2016).

Olteanu-Raimond et al. (2017) have recently undertaken a detailed review of the engagement of European NMAs with VGI. A survey was undertaken to elicit experiences with VGI, which revealed that few European NMAs are currently engaged with VGI and that those have developed their own VGI collection processes, mostly for change detection and the reporting of alerts, with less frequent examples of the reporting of new content, vernacular place names and photo interpretation (see Figure 1). In most cases the information gathered was

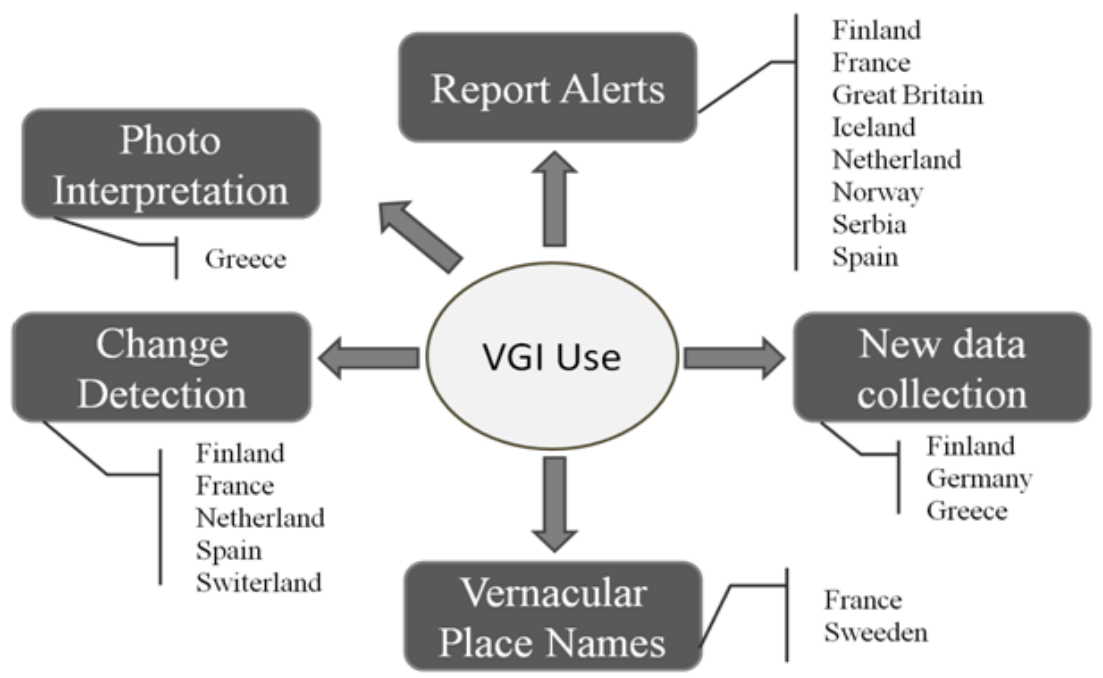

Fig. 1: Use of VGI by European NMAs. Source: Olteanu-Raimond et al. (2017). All rights reserved (C)ohn Wiley \& Sons Ltd. 
on traditional features included in standard topographic maps, such as roads, buildings and names. Very few mapping agencies have harvested and used the data collected by OSM or GeoNames.

The low involvement of European NMAs with VGI is related to five major barriers, which have been discussed in detail in Olteanu-Raimond et al. (2017); these are issues of data quality and validation; legal issues; issues related to the nature and motivation of the crowd; sustainability issues; and employment fears.

This chapter further develops the work of Olteanu-Raimond et al. (2017) by proposing a typical VGI collection workflow, which was considered by many NMAs such a good practice. This type of VGI platform is based on the main idea of a volunteered activity where contributors contribute directly to the platform by adding new features or attributes, correcting existing features, etc. It is important to mention that the integration of data coming from other crowdsourced activities, such as GPS traces from sports activities, are out of the scope of this chapter. The chapter is organised as follows: Section 2 focuses on the experiences of European NMAs with VGI by presenting some specific examples. Section 3 presents some recommendations for NMAs as a response to some of the five major barriers identified in the use of VGI. Finally, conclusions and future research directions are outlined in Section 4.

\section{Experiences with VGI}

As mentioned previously, most of the NMAs that engage with VGI have developed their own tools to collect data from citizens or from public partners. The aim of this section is to present an overview of some of these tools that completes and provides an update to the review reported in Olteanu-Raimond et al. (2017), which describes the experiences of NMAs in Finland, France, Greece, the UK, the Netherlands, Portugal and Switzerland, all of which responded positively to our call to contribute.

\subsection{Change Detection and Error Alerts}

Change detection and error alerts are among the most well developed VGI activities proposed by NMAs. Generally, alerts (e.g. to a new building or a new road name) are used as triggers to improve the quality of authoritative databases. The following outlines the experience of a series of NMAs in using VGI for change detection and error alerting.

At IGN France, change detection is generally undertaken by land surveyors who analyse a range of alert types and then contact local governments. Since 2008, IGN France has developed various applications that aim to report alerts concerning errors, change detection or vernacular toponyms (Viglino, 2009). 
These applications, deployed on different platforms and via different technologies (e.g. the Web, Android mobile phones and GIS) are mostly communitysourcing systems where professional partners, such as fire services and post offices, make reports on IGN data. A web application, accessible through the French Geoportal, was also developed for citizens, allowing them to make reports. These pioneering applications and their encouraging results have led the IGN to propose a unique community and citizen sourcing portal ${ }^{2}$, on which citizens can complete a form and provide location information, using GPS tracks, photographs or drawings, on an IGN basemap. A new version of the application is being tested that allows partners to access, add and modify features in an up-to-date copy of the topographic database. Contributions are first checked and validated by the surveyors with respect to data specifications, and quality expectations are checked by using quality indicators, visual checking and comparison with different data sources (e.g. construction permits issued by municipalities). Depending on the types of contributions, the VGI can be directly integrated into authoritative databases or used as a trigger for field work to improve the geometric precision of features.

With regard to future engagement with VGI, some research projects are currently under consideration. For example, Ivanovic et al. (2016) are studying the possibility of automatically inferring changes from additional sources found on the Web, including GPS tracks from hiking websites. The EU-funded Horizon 2020 LandSense project (2016-2020) will study the feasibility of updating Land Use/Land Cover (LULC) maps using Sentinel and in-situ citizen-derived data. Methods to aid quality assessment and conflict management in order to validate and integrate citizen-derived data into the authoritative database will also be explored (Leibovici et al., 2015).

In the Netherlands, Kadaster is running successful VGI activities, including 'terugmelding BRT' (alert on the Dutch Topographic Registry) and 'terugmelding BGT' (alert on the 'large scale' Topographic Registry), to report new changes and errors. Kadaster works as an open and transparent organisation, and contributors can easily see what has been done with their alerts. To stimulate and effectively motivate contributors, the staff working in the topographic department promptly validates all reported alerts. By directly updating the topographic maps when an error report is accepted, Kadaster shows its appreciation to the contributors and stimulates the further participation of citizens. In addition to the traditional data-updating by means of aerial and panoramic photographs, there is a growing tendency to use thematic data from external sources. The latter sources include governmental organisations, companies and also citizen contributors. In this context, Kadaster has proposed a second pilot (also known as the Sonneveld index) to collect data on religious buildings such as churches, mosques, synagogues, temples, monasteries and chapels; more than 1,000 addresses were collected by a group of enthusiastic contributors. As a result, Kadaster was able to enrich its topographic maps. 
Another VGI project was run to collect information on national border markers. On 30 October 1980, the Netherlands and Germany signed an agreement about the maintenance of the markers that define the borders between them; every three years, the national border markers must be inspected and, where necessary, maintained. In 2012, hikers were deployed to gather information about the situation of national border markers by using an ad hoc mobile application that also allows sending a picture. As a result, the Kadaster was able to make a decision as to whether it had to maintain a particular marker or not. The border markers application has recently completed its pilot phase, and a continuation of the project is being developed.

Finally, the forest paths project was a recent pilot based on VGI activities. In the Netherlands, the National Dutch Forest Organization (Staatsbosbeheer) is responsible for data on forests. The aim of the forest paths project was to use VGI to update the organisation's datasets. Kadaster provided raw material to forest rangers and asked them to verify and complete the map based on their field work. Kadaster has successfully completed pilot projects in Horsterwold and Flevopolder. The local forest rangers have updated their digital files on forest paths in their region. Kadaster is researching how to implement this method in the rest of the forested area in the Netherlands.

Ordnance Survey (OS), the NMA for Great Britain, has long engaged with customers and the general public for alerts about real-world change or errors reported in its paper or digital map products. While much contact is directly via telephone or written correspondence, a web map-based tool has been successfully trialled with public sector customers for reporting errors or omissions in a range of OS products. Using the 'Tell OS' interface, customers can locate, describe and submit their feedback for the product concerned. Their alerts are acknowledged and the information is fed into product management processes.

Sharing of volunteered information is also enabled for route-based information through the OS Maps application. Aimed at outdoor activities, the application enables the recording and sharing with other users of route information as part of its map display, search and navigation functionality.

\subsection{New-Feature Collection}

VGI provides the potential to capture new features or new information regarding existing features not previously collected by NMAs as it might not be within their mission priorities or it may be excluded for political or economic reasons.

In the Netherlands, Kadaster is running pilot projects to collect new features. One of these is the 'Crowdsourcing at school!' project, which is part of Kadaster's education programme. The aim of this initiative is to allow children to become familiar with VGI and with advancing society, but also to introduce them to the Kadaster organisation and its products and services. Children get a geographic orientation of the world in a playful way, and they also learn about 
their position within society. In this pilot, children collect data on emergency services such as police, ambulance and fire services. This project can also be used for data collection for other organisations or public services. The curriculum for this project is in a pilot phase and the first results have highlighted that VGI activities are not only for adults.

Linked to the large-scale renewal of Finland's National Topographic Database, a research project was launched by National Land Survey of Finland (NLS) at the beginning of 2016 to investigate the possibilities that VGI can offer in authoritative data collection. The project will build a concept to define the so-called 'Citizen's layer' to the authoritative topographic data, that is, a platform for data collection where they will be able to import or draw points, lines and polygons representing topographic objects in the real world. The concept will cover principles and tools for VGI data collection, e.g. for building up the service and the user interface as well as developing protocols and methods for engaging with citizens (Mooney et al., 2016). The quality and the best practices for using VGI will be identified in a pilot phase. The project seeks to validate data quality and usability and to investigate the possibilities of integrating VGI collected in the pilot to the authoritative database. As part of another research project, a hyper-local geosocial networking application (hylo.mygeotrust.org) was introduced for school children aged 14- to 15-years-old. With the mobile application, pupils were asked to map different kinds of objects in their neighbourhood to share their knowledge and observations. The initial results are encouraging. Children are interested in their local environments and have volunteered to map and share their knowledge on a map service. Based on these experiences, it seems beneficial to introduce the concept of a 'Citizen's layer' in schools as well.

Greek mapping authorities have been using VGI as a starting point to update or create new mapping outputs. The crowdsourced data are treated as an initial input layer that is compared against imagery backdrops (satellite or aerial). The VGI datasets are corrected, completed and re-assigned to the local nomenclature and then follow the normal processes for internally collected data.

Direção Geral do Território (DGT) is the NMA in Portugal; it coordinates Portugal's National Spatial Data Infrastructure (NSDI), SNIG, and develops research on geographic information. Presently, research on VGI at the DGT is focused on investigating how to use VGI in the production of official topographic data ${ }^{3}$. The general idea is to use case studies to demonstrate the potential benefits of including VGI as part of the authoritative database implementation strategy, benefits which include filling gaps in official data, enlarging the spatio-temporal coverage or addressing the aims of specific communities of interest. These benefits are in line with the more collaborative and participative approach presently adopted for SNIG development.. To identify and analyse the integration of VGI to NSDI, the environment and planning domain will be used as a target. Case studies will be designed to identify required modifications to the NSDI, such as changes to the metadata catalogue to accommodate 
VGI types or the interoperability and validation requirements for incorporating VGI within the NSDI. Moreover, a prototype based on a web service may become available through the NSDI geoportal, allowing any registered citizen to edit LULC polygons through the identification of geometry and/or classification changes. This will enable the analysis of thematic and positional inconsistencies reported by the users and define a strategy for including VGI in the production of official mapping.

Looking to future uses of VGI, the research interests of most of European NMAs range from motivational factors of volunteer engagement in VGI to change detection, data capture, and validation and management, all the way through to data or service delivery and associated quality and trust. In addition to VGI involving citizens, community groups and expert groups, exploring how VGI approaches might draw on the local knowledge of internal NMA employees is also of interest.

\subsection{Promoting the Usability of Authoritative Data}

In the past, within a research context, the Centro Nacional de Informação Geográfica (CNIG), which was then integrated in the Portuguese NMA (Instituto Geográfico Português (IGP), presently named Direção-Geral do Território (DGT) has been involved in the GEOCID (Hipólito et al., 2000) and Senses@ watch (Gouveia et al., 2004; Gouveia and Fonseca, 2008) projects, which represented early attempts to promote the involvement of citizens in the use or production of geographic information, and which shared some of the issues associated with the topic of VGI and its integration in an NSDI. The GEOCID project aimed to promote the use of SDI by citizens and represented a first effort to target citizens as users of these infrastructures, although it used a topdown approach (Fonseca and Gouveia, 2005). Senses@watch was a research project centred on the definition and evaluation of strategies to promote the use of environmental spatial information, such as water quality and noise, collected through citizens' senses (e.g. vision, hearing, taste and smell). A prototype of a Web-based collaborative site was developed, including an interface for mobile phones.

The results of these initial projects were successful, with a considerable level of citizen participation, but did not have the intended follow-up in the NMA services and workflows. Nevertheless, lessons could be learned from these experiences that can enrich present approaches to VGI. These projects enabled the confirmation of the SDI data user's increasing role and of the importance of providing participation at multiple levels where VGI can be seen as a resource for SDI. The assessment of the pragmatic implications of using ICT to support citizen participation in environmental monitoring or the identification of the major benefits of involving volunteer contributors (e.g. the promotion of public awareness on environmental issues; the cost-effectiveness of the method to maintain data collection activities; or the facilitation of the creation of early 
warning systems), as well as the corresponding drawbacks (e.g. the lack of data credibility), are just some of the insights about VGI provided by these activities.

\section{Recommendations for NMAs regarding VGI Use}

Starting with good/best practices identified in NMA experiences and research work, the goal of this section is to define recommendations for NMAs in organising a platform to collect and manage VGI. Compiling a list of expectations from both crowd or community sourcing and NMAs will ensure a fruitful relationship between both parties, as discussed by Olteanu-Raimond et al. (2017). From the NMA point of view, issues such as motivation, stability, consistency and minimisation of false entries are of concern, while feedback, the citizen layer and transparency for the crowd and community sourcing, among others, are some of the crowd's concerns. Here, we focus on six elements that are either barriers to the use of VGI or key elements that allow for the construction of a successful VGI platform for citizens, public and private partners and governments. The six elements are as follows: the data model and objects; the interface; motivation; identification; licensing; and quality control.

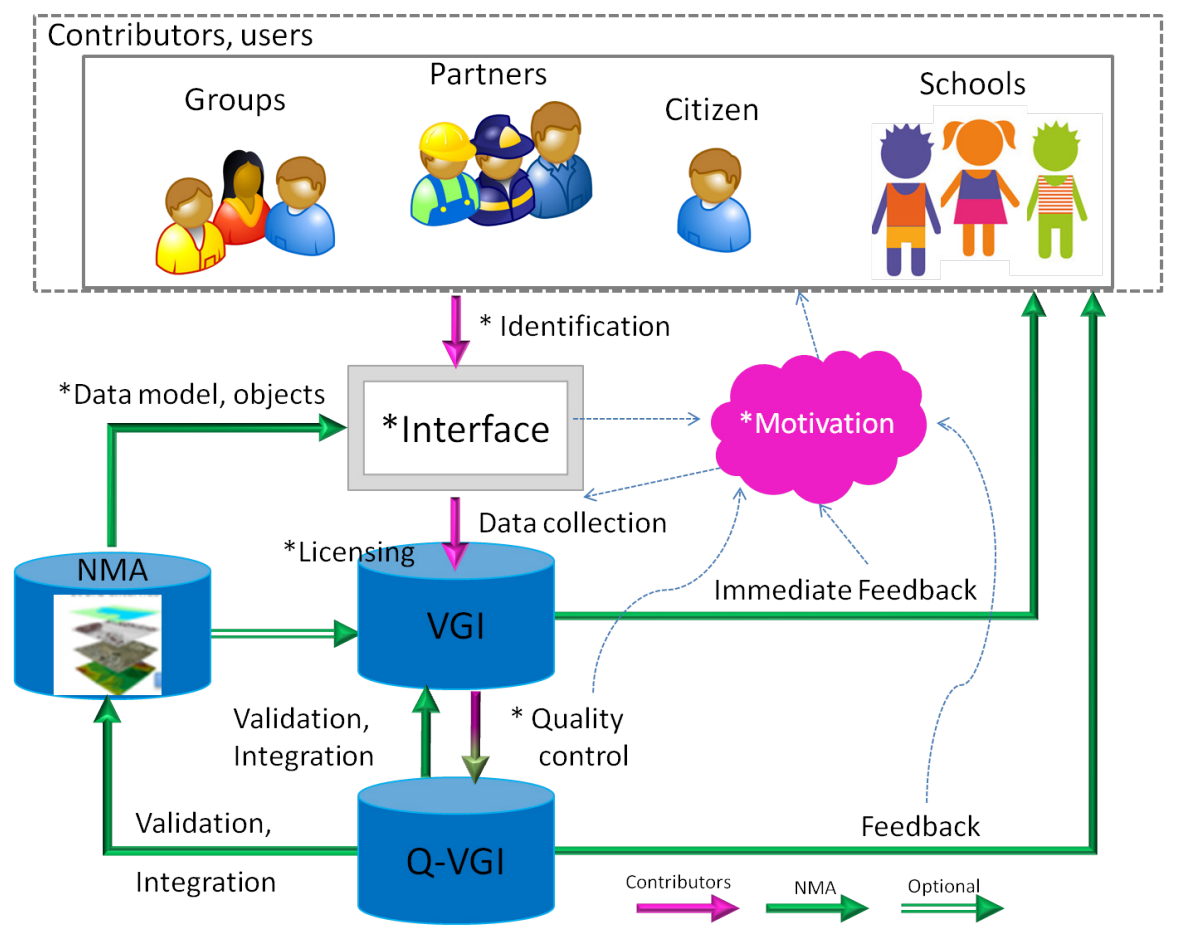

Fig. 2: A typical VGI collection workflow. 
A general workflow for VGI data collection is illustrated in Figure 2, where these six elements are marked with an asterisk $\left.{ }^{*}\right)$. In Figure 2, green and pink arrows represent NMA and contributor tasks, respectively.

A successful platform should be dedicated to both contributors and users, and should engage with citizens, specific groups of citizens sharing the same interest (e.g. hiking), partners (e.g. governments, emergency services) and the education community. Contributions should be made via user-friendly interfaces that implement an adaptive data model as proposed by NMAs, via secured identification, and via easy-to-use tools to contribute, manage, visualise and download VGI and/or authoritative data, depending on each NMA's data licence. A real added value from NMAs is the quality control of volunteered data, which can be corrected, validated and integrated into the VGI platform (Q-VGI to VGI). Depending on the data specification, some validated VGI can be integrated into the authoritative data (Q-VGI to NMA), in this way improving the accuracy and quality of the NMA's data. The quality control could be performed by contributors in a continuous way through the sharing of opinions on contributions, and step-by-step by the NMAs.

Table 1 summarises the recommendations described in the sections that follow and provides a list of opportunities and threats that can arise from such an NMA-VGI collection system. Opportunities and threats are described with respect to different elements identified in NMA data collection systems.

\subsection{Data Model and Objects}

Generally, NMAs are in charge of producing topographic databases by mapping the topography of the real world by focusing on specific types of objects described by few thematic features (e.g. number of lanes of a road, building type; Olteanu-Raimond et al., 2017). This implies that the existing features can be enriched by adding thematic information (e.g. number of floors in a building), but also that some new objects can be added. These new objects may be feature classes that are currently lacking in quality in official databases due to frequent real-world change (e.g. POIs, shops etc.), or data that can be most efficiently collected by contributors because collection is not feasible with remote mapping (e.g. hiking trails obscured by trees). Data that are of special interest to citizens or public services such as emergency services and municipalities, e.g. vernacular place names and traditional names of neighbourhoods (Castellote et al., 2013); obstacles, to help the navigation of people with disabilities (Rice et al., 2013); or paths, to improve pedestrian maps (Laakso et al., 2011) could be mapped by citizens having local knowledge, as suggested by Johnson and Sieber (2013).

\subsubsection{Citizen and Partner Layer}

Two of the identified barriers in using VGI are data quality and legal aspects (Olteanu-Raimond et al., 2017); Johnson and Sieber (2013) have reported the 


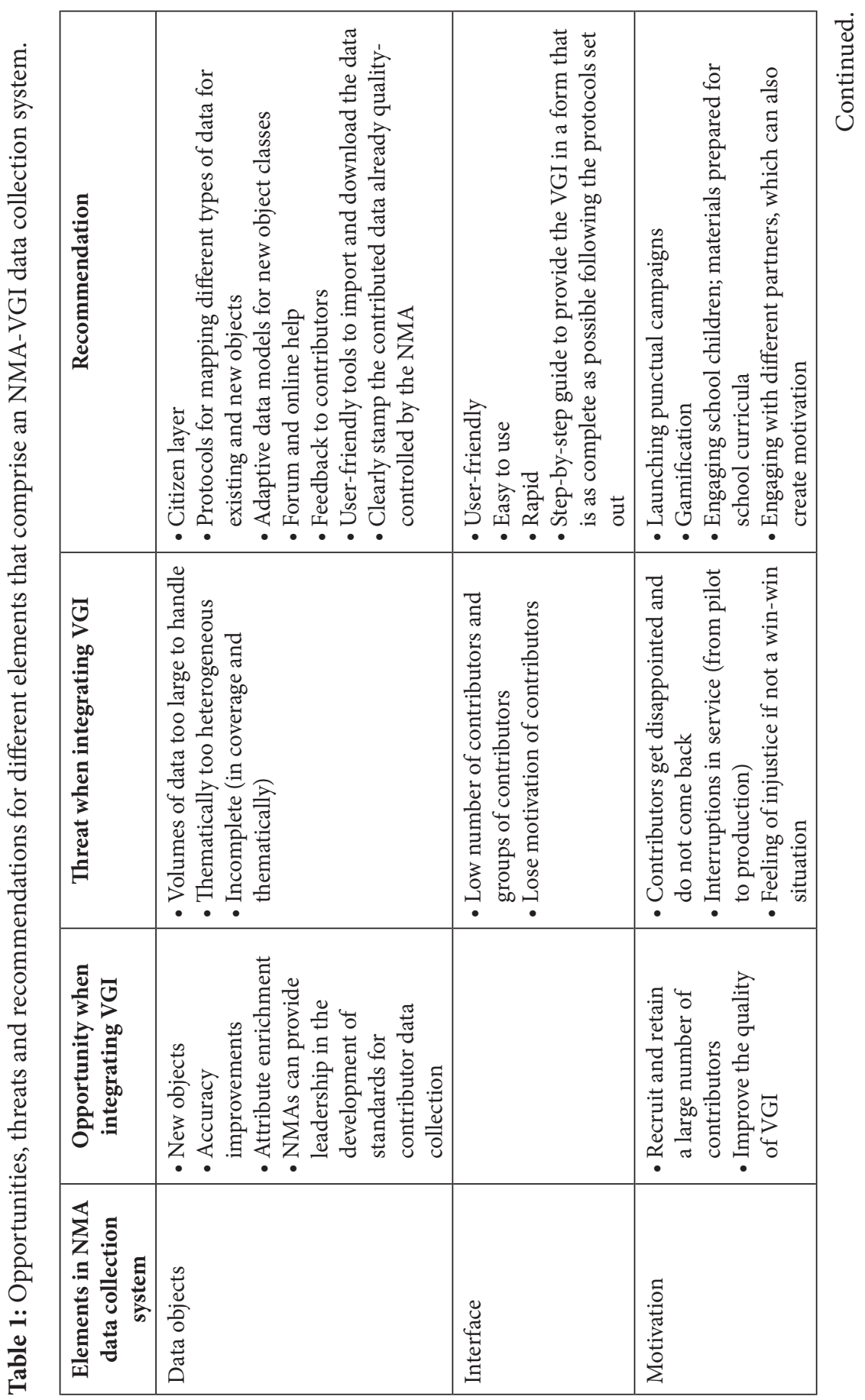




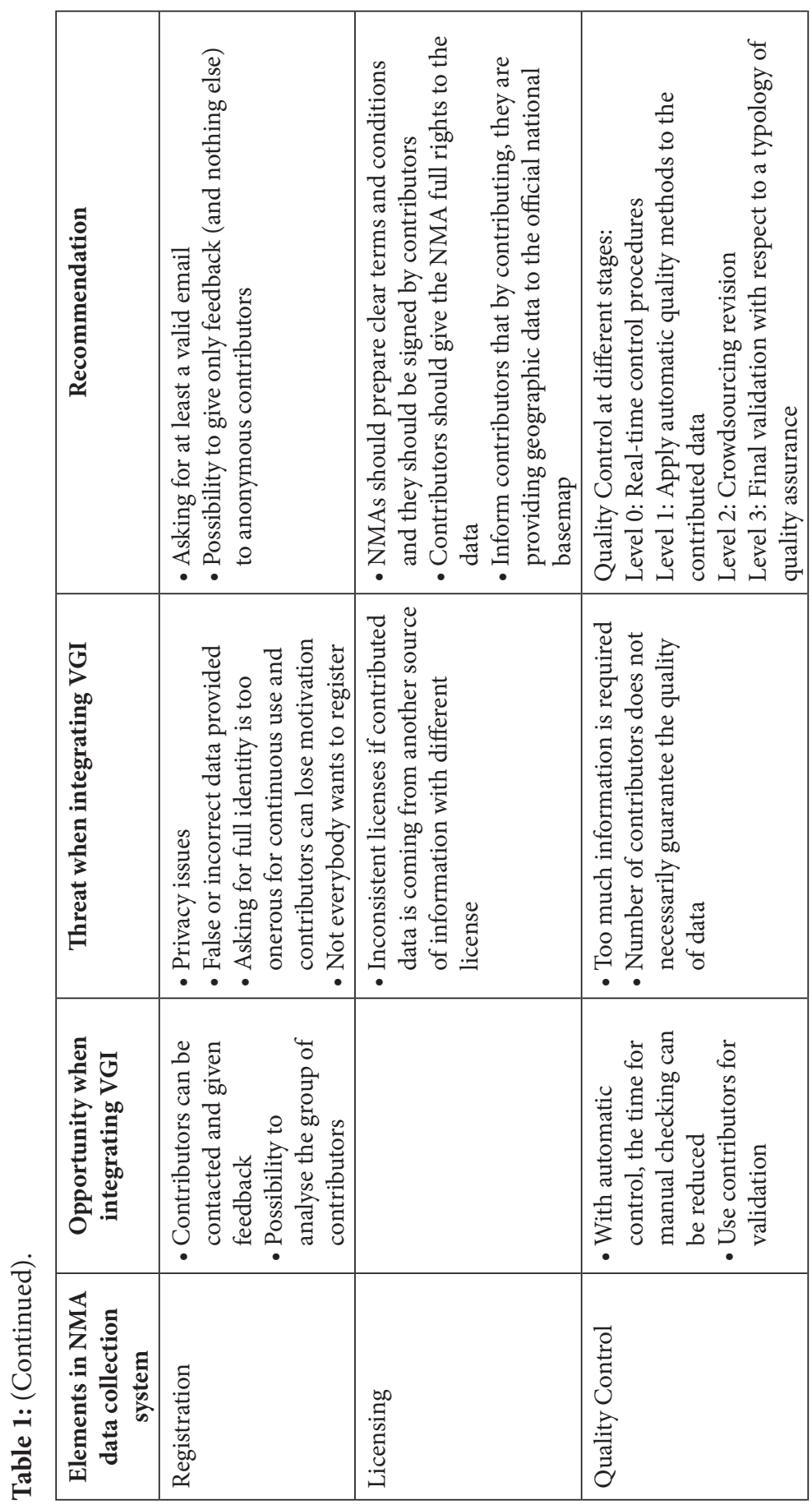


same finding regarding the use of VGI by governments and argued that a more formalised VGI collection process may prove beneficial. A solution to break down these barriers can be a participatory citizen and partner layer proposed by NMAs. In this way, the NMAs will first have the opportunity to add new content, but also to increase the usability of traditional topographic data, and, as a direct consequence, to improve the accuracy of the data and enrich the thematic information (e.g. 'the building is a private school with three entrances'). Then, NMAs can propose a formalised framework and standards, which would be expected by governments, to collect VGI with a focus on data validation, data quality assessment and integration methods, allowing the topographic data to be used to support other types of specific data (e.g. water pump locations for firefighters, billboard locations for local municipalities). We would make the following recommendations:

- Authoritative basemaps should be used to make contributions (e.g. topographic data, orthophotographs, satellite images, DEMs);

- NMAs should assess both the internal quality and the fitness-for-use of the volunteered data and should correct errors by using authoritative data as a topographic support; this could be a big added-value that would encourage different users, such as public authorities, security and emergency services, NGOs and citizens, to both contribute and use VGI;

- Volunteered data should be clearly stamped with quality-control stamps to easily distinguish between quality-controlled contributions and those not yet quality-controlled;

- Campaigns for specific data collection purposes should be organised;

- User-friendly tools to import and download data should be proposed to allow for a detailed selection of suitable data regarding spatiotemporal and social criteria and should be served to end users in different formats.

As mentioned in Section 2, the NLS of Finland has already decided to experiment with this new citizen layer concept through a research project.

\subsubsection{Adaptive Data Models for Object Collection}

NMAs should propose an adaptive data model to collect and monitor geographic objects. This data model should allow contributors to:

- report updates or errors;

- add new attributes and objects to existing class objects;

- add new class objects identified by NMAs as out of the specifications of the authoritative topographic databases at present, but important for different applications;

- add new class objects if it fits end user needs; 
- assess, as a mandatory requirement, the membership of an object to a class object type;

- import data collected in-situ; and

- ensure interoperability with the existing topographic data model in order to integrate significant and validated contributions directly into authoritative databases or to generate updates.

Nevertheless, proposing a quite open citizen and partner layer may introduce some threats, such as the possibility of obtaining large volumes of thematically heterogeneous data or data that are characterised by spatial and thematic incompleteness.

\subsubsection{Protocols for Object Collection}

The lack of protocols and the potential problems that this may entail, as well as recommendations for data collection, are discussed in more detail in Chapter 10 (Minghini et al., 2017). However, our additional recommendations regarding protocols are as follows:

- define a protocol for mapping different types of data for existing or new objects that balances the need to collect a minimum set of attributes and metadata with the desire for completeness in the data collection process;

- update and enrich the protocol regularly by taking into account end user experiences;

- propose a forum and online help facility to share experiences with and between contributors and assist contributors when needed; discussion forums have been proven to contribute, in some cases, to the creation of more reliable data (Haklay, 2010; Perger et al., 2012), and they are also a valuable tool for community building.

\subsubsection{Instant Feedback to Contributors}

From the different NMA experiences outlined above, it has been shown that engaging with contributors using transparent communication is crucial for a successful and sustainable platform. Good communication can be ensured by:

- one uniform feedback platform for the different products offered by the mapping agency: topographic data, citizen and partner layer, updates and error updates, etc.;

- contributor involvement in the feedback system building process;

- e-mails with updates concerning the status of contributions; and

- the display of contributor data immediately or in near real-time. 


\subsection{Interface}

Two kinds of user interface tools can be distinguished (Sabou et al., 2014): acquisition interfaces designed for and used by contributors to carry out crowdsourcing tasks, and management interfaces, which are required by the managers of the VGI project to monitor progress, assess quality and manage contributors. In this section we focus on the acquisition interface used by contributors for data collection, designated here as the contributor interface.

Switzerland's geoportal ${ }^{4}$ was recently awarded the '2015 eGovernment special prize ${ }^{5}$ at the ninth national eGovernment Symposium, which was held on 24 November 2015 in Bern involving representatives from the worlds of business, administration, politics and academia. Consistent use of open source software, open standards and cloud computing were the reasons for winning the prize. This geoportal features many properties that an NMA contributor interface should provide (e.g. an intuitive and contributor-friendly interface, a VGI component with the recently renewed revision service with immediate customer feedback, and a smooth, dynamic and interactive map navigation) a report option for customer alerts and the use of open standards. Our recommendations for the contributor-interface are as follows:

- offer a contributor-friendly interface that guides the contributor to supply all the information required by the protocols (e.g. metadata, attributes);

- define contributor-friendly interfaces that incorporate the NMA protocols and best practices without negatively impacting upon contributor enthusiasm or hampering the flow of data;

- provide tools to support the training of contributors and/or groups of contributors according to the goal;

- through the interfaces for collaboration, address the need to educate contributors and easily integrate the non-traditional types of data that they might collect;

- whenever possible, make use of standard, interoperable data formats and services in order to further extend the interface and/or integrate new services and applications;

- use up-to-date basemaps and do not overload the platform with multiple themes;

- adequately accommodate all the VGI types, which are of a diverse nature, dynamic, and sometimes produced in real-time;

- implement the full editing of objects rather than just hints attached loosely to existing data objects.

Additionally to what has been mentioned above, an intuitive contributor-interface can also play another very important role in the field of input data quality. While the basic principles of human-computer interaction (HCI) should be 
intact and meticulously followed, the contributor-interface could be the vehicle for implementing a number of elements in the protocol regarding the input of high-quality data (more information protocols for data capture can be found in Chapter 10 by Minghini et al., 2017). It is common for NMAs to have protocols in place that must be followed in order to achieve maximum homogeneity in the datasets produced. Volunteered content should also follow similar rules. Thus, the contributor-interface, which in this case serves as the data capturing layer, should be equipped with as many protocol elements as possible, balancing between high data integrity (and thus quality) and adequate freedom for the contributor.

\subsection{Motivation}

An important part in the success of using VGI is engaging people. Interested readers will find a detailed discussion on user motivation and engagement in Chapter 5 (Fritz et al., 2017). NMA experiences have shown that citizens are often not really interested in getting paid or in being presented with awards or prizes: having the possibility to contribute geographic information from their personal surroundings with a direct impact on publicly visible maps and getting feedback from NMAs are the main positive reasons to contribute. Nevertheless, in order to increase the number of contributors and ensure sustainability, NMAs should first promote, advertise and permeate the crowds, and secondly motivate, activate and reward contributions. However, when implementing reward systems, these rewards should not encourage contributors to favour quantity over quality in their contributions.

\subsubsection{Gamification Techniques}

Undoubtedly, a contributor-interface that enhances contributor experience can help to engage contributors; however, this factor alone is not enough to create the drivers and support the motivation that need to be achieved to attract a large pool of contributors to an initiative. There are a number of research efforts around the use of gamification techniques (Antoniou and Schlieder, 2014; Yanenko and Schlieder, 2014) to achieve these levels of motivation. Gamification, loosely defined, is the implementation of gaming practices in a non-game context. In essence, gamification, through game mechanics and game design, can have an impact and influence on participant behaviour. The aim of gamification is to make the participant achieve certain goals by enhancing engagement, improving performance and multiplying participation efforts towards a goal. Thus, NMAs can considerably enhance citizen motivation by implementing gamification processes for data capturing or change detection. 


\subsubsection{Giving Feedback}

Feedback to contributors, given by sending updates concerning the status of their individual or group contributions, is an important motivation for contributors. Organisations need to assess the likelihood of such motivations being strong enough in a prospective contributor community to ensure the sustainability of their proposed VGI initiative (Hickling Arthurs Low Corporation, 2012). To help sustain contributions over time, some recommendations are listed here:

- all contributions should be welcome (e.g. attributes such as 'gravel road now paved' can be as valuable as topographic data);

- contributors want to receive acknowledgement for their contributions and to get rapid evidence that these contributions have been used;

- the process of making contributions should be as easy and streamlined as possible, as contributors may not be strongly motivated to contribute to extensive feature classification and the metadata requirements of public mapping programs; and

- different contributor-interfaces may be required for first-time or occasional contributors than for internal production staff or external power contributors; for example, tools that allow inappropriate content to be reported through a link allow contributors some control over data quality

- (Coleman, 2010; Esri, 2010; Hickling Arthurs Low Corporation, 2012).

\subsubsection{Engage with Groups of Users}

A number of advertising activities can be used to attract contributors to a VGI project. In a study on the impact of contributors to VGI projects (Schmidt et al., 2012), it is proposed to attract diverse groups of contributors with projectrelated mapping, to make mapping easy for beginners and to keep contributors mapping with social mapping events, as typically happens with OSM (Mooney et al., 2015). Launching campaigns will attract a number of users for a time period, whereas connecting relevant user groups (e.g. land owners having an interest in maintaining boundaries) will create more devoted contributors. In general, people who use the data will feel more attached to the project and will be more willing to contribute. In addition, it will be easier for them to find possible errors and report/make corrections if the procedures are made as easy as possible. Campaigns should target groups such as landowners, school children, cyclists, joggers, scouts, orienteering enthusiasts, hunters, hikers and geocachers, among others, who may be more willing to contribute due to their special interests and because they will take personal advantage of the addition of the VGI to the NMA database. 


\subsubsection{Engage with Public Partners}

Some strategic public partners may be very important for the collection of certain types of data. For example, municipalities can easily engage with citizens for urban planning purposes and security-related partners that manage emergencies, such as civil protection authorities or firefighters, who are very often in the field and have specific needs such as fire hydrants, obstacles, building entrances, etc.

\subsubsection{Engage with Schools}

Introducing the work of the NMA and the idea and principles of topographic data collection to school pupils may be a good way to disseminate knowledge and could shape a large number of future contributors. To put this idea into practice, the following recommendations can be given:

- the collection of data must be integrated within an education programme (i.e. teaching by collecting data);

- close cooperation with teachers is crucial: teachers are busy with their everyday work, so they need to have some ready, easy-to-adapt teaching materials;

- school pupils are a very motivated group, but an application for data collection by pupils must work perfectly and rapidly;

- before data collection starts, it is important to explain to the pupils what crowdsourcing is and how it works; pupils need to understand that they are an important part of society and that they can deliver valuable data for others;

- data collection projects for pupils need to have some fun aspects that reward pupils who deliver good-quality data, which can even further support their motivation - gamification is one possible approach that may fit well with the needs of this particular group; and

- different stakeholder groups (pupils, parents, teachers, etc.) should be invited to refine/improve the curriculum.

Although unrelated to VGI data collection for NMAs, two successful examples of the engagement of pupils that have taken the above recommendations into account are described in Brovelli et al. (2016) and Ebrahim et al. (2016).

\subsection{Registration}

Data contributors may be anonymous, but this may permit vandalism (e.g. mapping fake features or deleting features that exist) and the contribution of 
fraudulent data or spam. It is still not entirely possible to distinguish between a credible VGI contributor on the one hand and an incompetent one, a mischiefmaker or an outright vandal on the other hand (Coleman, 2010), although research is ongoing in this area: for example, Ciepłuch et al. (2010) have studied the history and the profiling of contributors; Van Exel et al. (2010) have proposed the experience, recognition and local knowledge of the individual as an indicator of quality input; and D'Antonio et al. (2014) have proposed an evaluation model for the contributor's reputation and data trustworthiness. However, based on the NMAs' experiences, very few bad contributions have been spotted, and in general more than $80-90 \%$ of the citizen contributions are useful (Olteanu-Raimond et al., 2017). Registered contributors are expected to have a more consistent contribution, since participating in the registration process proves their motivation and their intention to be identified. Apart from the contributor identification, registration has additional advantages (e.g. the contribution can be saved and finalised later by, for instance, tagging the position in the field and submitting the contribution later by using a computer at home). Three different types of profiles (see Table 2) could be made available when contributors register depending on the type of organisational model for data collection used and the validation process applied by the NMA afterwards; these include:

- Strong registration: this may create more powerful contributors in terms of permitted activities with the data. Full identity should be required in sensitive cases such as cadastral information for property owners. Another category may be a contract contributor (other authorities, for example) with specific permissions but also contribution obligations.

- Light registration: this type of identification allows the organisation collecting the data to contact the contributor if needed and to learn more about contributors, e.g. to determine potentially useful information such as their field of expertise.

- Weak registration: this only requires a valid email and password for registration to create a user account.

Table 2: Types of contributor registration profiles.

\begin{tabular}{|l|l|l|}
\hline \multicolumn{1}{|c|}{ Strong registration } & Light registration & Weak registration \\
\hline $\begin{array}{l}\text { Full identity, e.g. from e-government } \\
\text { authentication systems, such as: }\end{array}$ & Valid email & Valid email \\
full name, full address or postcode, & Profession & Password \\
profession and institution, phone & Age / age group & \\
number, passport/ID number & Gender & \\
\hline
\end{tabular}




\subsection{Quality Control}

In Chapter 7 (Fonte et al., 2017), an overview of the quality indicators that can be used to assess VGI is presented. Traditional spatial data quality assessment measures can be used. These can be applied using reference data, such as control data provided by experts, or through the comparison of data coming from several sources, which may even be VGI, enabling the assessment of logical consistency. Additionally, other indicators can be used to assess the reliability of the data, such as metadata on the data acquisition procedure, indicators about the contributor, socio-economic indicators or the consistency of corresponding data with different origins.

The quality control could be carried out at different levels that aim to facilitate the final validation by the NMA (which is mandatory), as outlined in the following subsections.

\subsubsection{Level 0: Real-time Control Procedures}

This initial level of quality control ensures that the minimum required information specified in the data collection protocol is provided and that no inconsistencies are introduced. It aims to assist the contributor in mapping valid information and is performed during the collection phase. Note that the absence of inconsistencies does not imply that the data are accurate and reliable. It controls:

- Required metadata and attributes. If the minimum information required by the protocols is not provided, alerts to the contributors asking for additional information should be sent. The submission of a new contribution can be approved once this control check is successful, i.e. the contributor can then submit the data. Care should be taken to minimise the mandatory information needed to avoid negatively impacting contributor motivation.

- Logical consistency. Automatic rules implementing some basic topological consistency checks (e.g. a polygon must be closed, roads should be topologically related, etc.) should be applied in real-time when a contribution is submitted. The system should recognise the presence of some types of inconsistencies in contributions and then not allow the submission of these contributions, such as in the case of clear topological mistakes; in other cases, the system should not prohibit submission but generate warning messages to the contributors suggesting corrections or additional checks, as contributors may in fact be providing important information about significant changes in the terrain. 


\subsubsection{Level 1: Applying Automatic Quality Control Methods to the Volunteered Data}

The goal of Level 1 checks concerns data quality assessment through applying automatic methods. Three approaches are recommended:

- Data reliability. Automatic procedures can be used to perform an initial check of data reliability. Several sources of VGI can be used to assess data agreement: this refers to comparison of corresponding positional and attribute information, such as the position of roads in different data sources. The logical consistency of contributions can also be used to assess their reliability; for example, if a building is positioned inside a lake, the information may be considered to have low reliability.

- Contributor-based data reliability. If a prior assessment of contributor reliability is performed, for example by maintaining historical data on the contributors, it is possible to associate a degree of reliability to the data that is related to the reliability of the contributor.

- Specification-based reliability. Reliability of contributions can also be assessed by considering NMA specifications associated with the object being contributed. For example, if a building with an area lower than the NMA specification for the minimum size of buildings is mapped, this contribution can be automatically tagged as not fit-for-purpose.

\subsubsection{Level 2: Crowdsourcing Revision}

Crowdsourcing revision consists of:

- In-situ campaigns. Mapping agencies can organise in-situ campaigns asking contributors to assist in the validation of some highlighted complex cases where the NMA has insufficient information to perform a final validation. An example of this can be the assignment of a land cover class to particular locations when no field visits have been made.

- Peer validation between contributors. The VGI platform should provide additional capabilities to enable contributors to vote on ('thumbs up' or 'down') or to comment and discuss contributions. Discussions and/or comments can generate new insights into the main difficulties, and eventually some reliability indicators can be associated with specific types of contributions or to some areas, e.g. the classification of certain land cover types may be difficult for contributors, or contributions from a particular area can be found to have different interpretations. These indicators can be useful for assessing data reliability. 


\subsubsection{Level 3: Final Validation with Respect to a Typology of Quality Assurance}

Methods to visualise quality, discussed in Chapter 9 (Skopeliti et al., 2017), can considerably enhance this step in the process. Some recommendations include:

- Define a typology of quality and associate the indicators previously assessed to either qualitative or quantitative rankings; these rankings can be based on probabilistic, fuzzy or possibilistic approaches.

- To optimise the use of resources and procedures, NMAs may perform validation only on volunteered data that are considered to be worth validating depending on the indicators obtained in the previous step that are considered relevant for each dataset.

- Final decisions are taken on the quality of the contributions and their usefulness, depending on the quality values obtained with the adopted quality typology.

- As the final validation assesses how good the contributor input was, this information may be used to rank contributor performance.

\subsection{Licensing}

With VGI, an important issue arises regarding the intellectual property of the data, which should be handled through licensing and consent. Contributors should give the NMA full rights to the data so that the NMA can take full advantage of the contributed data; this consent can be obtained either during the registration phase or after the first contribution is made. The contributors should be informed that by contributing, they are providing geographic data to the official national basemap. A well defined licence for the NMA's sharing and use of geographic data should be provided to and agreed upon by the contributors.

Some other legal aspects, such as liability and privacy, can differ from country to country or from product to product. These aspects are discussed in more detail in Chapter 6 (Mooney et al., 2017).

\section{Conclusion}

In this chapter, a review of the different VGI experiences of a few European NMAs was presented, and guidelines and recommendations were presented to help mapping agencies better exploit the opportunities offered by VGI through volunteered activities made by contributors.

Due to its nature and characteristics, VGI is still seen by NMAs, and more generally by government bodies, as having low quality and as a source of unreliable data. Therefore, few NMAs are engaged with VGI. When they are engaged, they have generally proposed their own tools to collect reports, and only rarely 
has VGI been used to collect data on features beyond the standard set mapped by NMAs.

Even though this type of data needs the development of new and different procedures for collection (see Chapter 10 by Minghini et al., 2017) or quality assessment (see Chapter 7 by Fonte et al., 2017) to become of major interest, VGI is nevertheless a valuable source of data, as it may help NMAs to provide data that are more up-to-date as well as to collect new, additional data that better address user needs. New features usually not collected by NMAs, either due to cost restrictions or because they represent non-traditional topographic data, could be of value to citizens and to various public services and government agencies.

To engage with the VGI community, the main recommendation for an NMA is to build a VGI platform that allows users to make reports but also to collect new, additional features that are not traditionally collected, to create a citizen and partner layer. An increasing number of VGI projects to collect data have been proposed during the last decade. As noted in the review by See et al. (2016), there is considerable variability in both the sustainability and the goal of the VGI projects. Some of them have been successfully operating for a long time while others have a finite life, being linked to some specific events, or are no longer active or available online. Moreover, few governments and municipalities have proposed platforms to collect data from citizens for purposes such as urban planning. Other public services, such as medical emergency departments or fire services, use their own resources to collect specific spatial data (e.g. water pumps, obstacles, building entrances), which need to be matched to spatial reference data.

Being aware of these current practices and initiatives, the question of why an NMA should also propose a VGI platform is a relevant one. We believe that NMAs, as public bodies, on the one hand are officially responsible for providing accurate and reliable information through SDIs to all potential users and, on the other hand, have the necessary expertise to manage and integrate spatial data. Moreover, all of the public initiatives mentioned earlier could not be implemented without important financial and human resources for deploying the GIS systems to collect, manage and maintain data and to train agents to deal with spatial information. We believe that a stronger collaboration between NMAs and governments through a VGI platform could result in a public-cost reduction and a better service to citizens, where these could be more involved in decision-making or in supporting security issues that affect their lives in a positive or negative way. Thus, for a successful VGI platform, one of the most important recommendations is to engage with citizens in general, specific groups of citizens having the same interests, and groups of public and governmental bodies, including the educational system. Engaging with different public bodies and with the educational sector will increase citizen involvement since these bodies are close to citizens and may invest in the future by educating and raising the awareness of younger generations regarding the relevance 
of spatial data and their quality. These engagements could create motivation, increase sustainability and promote good-quality data for both NMAs and the contributors.

Another important aspect, more oriented towards citizens, that can increase motivation is gamification. However, when implementing reward systems (of gamified or real-life rewards), attention should be paid to the fact that data quality is much more important than quantity, and this should be clearly explained to the contributor. Thus, a good practice for gamification is to avoid giving rewards or prizes based on (or only on) the number of contributions made.

We feel that a platform based on the recommendations discussed in this chapter is feasible and can be carried out in a step-by-step manner through the development of pilots and research projects, as exemplified by the ongoing initiative of the Finnish mapping agency, which is defining and preparing to test the concept of a citizen layer.

Due to the importance of and increasing trend in VGI, we believe that NMAs should develop national VGI platforms for both data collection and data dissemination, even if it is difficult to predict if, or when, these initiatives will really become a 'standard practice' for all NMAs.

\section{Acknowledgements}

The authors would like to thank swisstopo for hosting the WG3 meeting that initiated the discussion on recommendations for NMAs involved in activities with VGI. We would also like to thank the different participants who contributed to the discussions and are not authors of this chapter: Bianka Fohgrub, Jasper Hogerwerf, Oana Popescu, Jean-Christophe Guélat, André Streilein and Peter Mooney. Finally, we are grateful to the referees for their helpful comments on the original version of this chapter.

\section{Previous publication}

Figure 1 has been previously published in Olteanu-Raimond, A.-M., Hart, G., Foody, G., Touya, G., Kellenberger, T., Demetriou, D., 2017. The scale of VGI in map production: A perspective of European National Mapping Agencies. Transactions in GIS 21, 74-90. DOI: https://doi.org/10.1111/tgis.12189 and is being reproduced here with permission from John Wiley \& Sons Ltd.

\section{Notes}

${ }^{1}$ https://www.gov.uk/government/publications/open-data-charter/ g8-open-data-charter-and-technical-annex, published in June 2013

${ }^{2}$ https://espacecollaboratif.ign.fr/ 
${ }^{3}$ http://snig.dgterritorio.pt/portal/

${ }^{4}$ http://www.geo.admin.ch

${ }^{5}$ https://www.news.admin.ch/message/index.html?lang=en\&msg-id=59628

\section{Reference list}

Antoniou, V., Morley, J., Haklay, M., 2009. The role of user generated spatial content in mapping agencies, in: Proceedings of GIS Research UK $19^{\text {th }}$ Annual conference, Durham, UK, 1-3 April 2009, pp. 251-255.

Antoniou, V., Schlieder, C., 2014. Participation patterns, VGI and gamification, in: Proceedings of AGILE 2014. Presented at the AGILE 2014, Castellón, Spain, pp. 3-6.

Brovelli, M.A., Minghini, M., Molinari, M., Molteni, M., 2016. Do open geodata actually have the quality they declare? The case study of Milan, Italy, in: International Archives of the Photogrammetry, Remote Sensing and Spatial Information Sciences. Prague, Czech Republic.

Castellote, J., Huerta, J., Pescador, J., Brown, M., 2013. Towns conquer: a gamified application to collect geographical names (vernacular names/ toponyms). Presented at the AGILE 2013, Leuven, Belgium, 14-17 May 2013. Available from: https://agile-online.org/Conference_Paper/CDs/ agile_2013/Short_Papers/SP_S2.3_Castellote.pdf [Last accessed 16 May 2017].

Ciepłuch, B., Jacob, R., Mooney, P., Winstanley, A., 2010. Comparison of the accuracy of OpenStreetMap for Ireland with Google Maps and Bing Maps, in: Proceedings of the Ninth International Symposium on Spatial Accuracy Assessment in Natural Resources and Environmental Sciences, Leicester, UK, 20-23 July 2010, pp. 337-340. Available from: http://www.spatial-accuracy. org/system/files/img-X07133419_0.pdf [Last accessed 16 May 2017].

Coleman, D., 2010. Volunteered geographic information in spatial data infrastructure: An early look at opportunities and constraints, in: Rajabifard, A., Crompvoets, J., Kanantari, M., Kok, B. (Eds.), Spatially Enabling Society: Research, Emerging Trends and Critical Assessment. Leuven University Press, Leuven, Belgium, pp. 1-18.

D’Antonio, F., Fogliaroni, P., Kauppinen, T., 2014. VGI edit history reveals data trustworthiness and user reputation, in: Proceedings of AGILE 2014, Huerta, J., Schade, S., Granell, C. (Eds.), Castellón, Spain, 3-6 June 2014. Available from: https://agile-online.org/Conference_Paper/cds/agile_2014/ agile2014_140.pdf [Last accessed 16 May 2017].

Ebrahim, M., Minghini, M., Molinari, M., Torrebruno, A., 2016. Minimapathon: mapping the world at 10 years old, in: Proceedings of the 8th Annual International Conference on Education and New Learning Technologies (EDULEARN 2016). Presented at the 8th Annual International Conference on Education and New Learning Technologies (EDULEARN 2016), Barcelona, 
Spain, 4-6 July 2016, pp. 4200-4208. DOI: https://doi.org/10.21125/edulearn.2016.2018

Esri, 2010. Simplifying Citizen Reporting. ArcUser Online. Available at http://www. esri.com/news/arcuser/0111/citysourced.html [Last accessed 1 May 2017].

Fonseca, A., Gouveia, C., 2005. Spatial multimedia for environmental planning and management, in: Campagna, M. (Ed.), GIS for Sustainable Development. CRC Press, pp. 143-165.

Fonte, C C, Antoniou, V, Bastin, L, Estima, J, Arsanjani, J J, Bayas, J-C L, See, L and Vatseva, R. 2017. Assessing VGI Data Quality. In: Foody, G, See, L, Fritz, S, Mooney, P, Olteanu-Raimond, A-M, Fonte, C C and Antoniou, V. (eds.) Mapping and the Citizen Sensor. Pp. 137-163. London: Ubiquity Press. DOI: https://doi.org/10.5334/bbf.g.

Fritz, S, See, L and Brovelli, M. 2017. Motivating and Sustaining Participation in VGI. In: Foody, G, See, L, Fritz, S, Mooney, P, Olteanu-Raimond, A-M, Fonte, C C and Antoniou, V. (eds.) Mapping and the Citizen Sensor. Pp. 93-117. London: Ubiquity Press. DOI: https://doi.org/10.5334/bbf.e.

Goodchild, M.F., 2007. Citizens as sensors: the world of volunteered geography. GeoJournal 69, 211-221. DOI: https://doi.org/10.1007/s10708-007-9111-y

Gouveia, C., Fonseca, A., 2008. New approaches to environmental monitoring: the use of ICT to explore volunteered geographic information. GeoJournal 72, 185-197. DOI: https://doi.org/10.1007/s10708-008-9183-3

Gouveia, C., Fonseca, A., Câmara, A., Ferreira, F., 2004. Promoting the use of environmental data collected by concerned citizens through information and communication technologies. Journal of Environmental Management 71, 135-154. DOI: https://doi.org/10.1016/j.jenvman.2004.01.009

Haklay, M., 2010. How good is volunteered geographical information? A comparative study of OpenStreetMap and Ordnance Survey datasets. Environment and Planning B: Planning and Design 37, 682-703. DOI: https://doi. org/10.1068/b35097

Hickling Arthurs Low Corporation, 2012. Volunteered Geographic Information (VGI) Primer. Natural Resources Canada. Available from: http://ftp. maps.canada.ca/pub/nrcan_rncan/publications/ess_sst/291/291948/cgdi_ ip_21e.pdf [Last accessed 16 May 2017].

Hipólito, J., Fonseca, A., Gouveia, C., Fava, S., Henriques, R.G., Neves, J.N., 2000. GEOCID: Implementation of a National Geographic Information Infrastructure for the Citizen, in: The 6th EC and GIS Workshop - The Spatial Information Society - Shaping the Future, Lyon, France, 28-30 June 2000. Available from: http://www.ec-gis.org/Workshops/6ec-gis/papers/ hipolito-geocid.doc [Last accessed 16 May 2017].

Ivanovic, S., Olteanu-Raimond, A.-M., Mustière, S., Devogele, T., 2016. Detection of outliers in crowdsourced GPS traces, in: Bailly, J.-S., Griffith, D., Josselin, D. (eds.), Proceedings of Spatial Accuracy 2016, Montpellier, France, 5-8 July 2016, pp.130-135. Available from: http://www.spatial-accuracy. org/system/files/Detection\%20of\%20outliers\%20in\%20crowdsourced $\% 20$ GPS\%20traces.pdf [Last accessed 16 May 2017]. 
Johnson, P., Sieber, R., 2013. Situating the adoption of VGI by government, in: Sui, D., Elwood, S., Goodchild, M. (Eds.), Crowdsourcing Geographic Knowledge. Springer Netherlands, pp. 65-81.

Karimipour, F., Azari, O., 2015. Citizens as expert sensors: One step up on the VGI ladder, in: Gartner, G., Huang, H. (Eds.), Progress in Location-Based Services 2014. Springer International Publishing, Cham, pp. 213-222.

Laakso, M., Sarjakoski, T., Sarjakoski, L.T., 2011. Improving accessibility information in pedestrian maps and databases. Cartographica: The International Journal for Geographic Information and Geovisualization 46, 101-108. DOI: https://doi.org/10.3138/carto.46.2.101

Leibovici, D.G., Evans, B., Hodges, C., Wiemann, S., Meek, S., Rosser, J., Jackson, M., 2015. On data quality assurance and conflation entanglement in crowdsourcing for environmental studies, in: ISPRS Annals of the Photogrammetry, Remote Sensing and Spatial Information Sciences, Volume II-3/ W5, La Grande Motte, France, 28 September to 3 October 2015, pp. 195202. Available from: http://www.isprs-ann-photogramm-remote-sensspatial-inf-sci.net/II-3-W5/195/2015/isprsannals-II-3-W5-195-2015. pdf. DOI: https://doi.org/10.5194/isprsannals-II-3-W5-195-2015 [Last accessed 16 May 2017].

Minghini, M, Antoniou, V, Fonte, C C, Estima, J, Olteanu-Raimond, A-M, See, L, Laakso, M, Skopeliti, A, Mooney, P, Arsanjani, J J, Lupia, F. 2017. The Relevance of Protocols for VGI Collection. In: Foody, G, See, L, Fritz, S, Mooney, P, Olteanu-Raimond, A-M, Fonte, C C and Antoniou, V. (eds.) Mapping and the Citizen Sensor. Pp. 223-247. London: Ubiquity Press. DOI: https://doi.org/10.5334/bbf.j.

Mooney, P., Minghini, M., Laakso, M., Antoniou, V., Olteanu-Raimond, A.-M., Skopeliti, A., 2016. Towards a protocol for the collection of VGI vector data. International Journal of Geographic Information 5 (11), 217. DOI: https:// doi.org/10.3390/ijgi5110217

Mooney, P., Minghini, M., Stanley-Jones, F., 2015. Observations on an OpenStreetMap mapping party organised as a social event during an open source GIS conference. International Journal of Spatial Data Infrastructures Research 10, 138-150. DOI: https://doi.org/10.2902/ijsdir.v10i0.395

Mooney, P, Olteanu-Raimond, A-M, Touya, G, Juul, N, Alvanides, S and Kerle, N. 2017. Considerations of Privacy, Ethics and Legal Issues in Volunteered Geographic Information. In: Foody, G, See, L, Fritz, S, Mooney, P, Olteanu-Raimond, A-M, Fonte, C C and Antoniou, V. (eds.) Mapping and the Citizen Sensor. Pp. 119-135. London: Ubiquity Press. DOI: https://doi. org/10.5334/bbf.f.

Olteanu-Raimond, A.-M., Hart, G., Foody, G., Touya, G., Kellenberger, T., Demetriou, D., 2017. The scale of VGI in map production: A perspective of European National Mapping Agencies. Transactions in GIS 21, 74-90. DOI: https://doi.org/10.1111/tgis.12189

Perger, C., Fritz, S., See, L., Schill, C., van der Velde, M., McCallum, I., Obersteiner, M., 2012. A campaign to collect volunteered geographic infor- 
mation on land cover and human impact, in: Jekel, T., Car, A., Strobl, J., Griesebner, G. (Eds.), GI_Forum 2012: Geovisualisation, Society and Learning. Herbert Wichmann Verlag, Berlin / Offenbach, pp. 83-91.

Rice, M.T., Jacobson, R.D., Caldwell, D.R., McDermott, S.D., Paez, F.I., Aburizaiza, A.O., Curtin, K.M., Stefanidis, A., Qin, H., 2013. Crowdsourcing techniques for augmenting traditional accessibility maps with transitory obstacle information. Cartography and Geographic Information Science 40, 210-219. DOI: https://doi.org/10.1080/15230406.2013.799737

Sabou, R., Bontcheva, K., Derczynski, L., Scharl, A., 2014. Corpus annotation through crowdsourcing: Towards best practice guidelines. Presented at the LREC 2014, Ninth International Conference on Language Resources and Evaluation, European Language Resources Association (ELRA), Reykjavik, Iceland.

Schmidt, M., Klettner, S., Steinmann, R., Häusler, E., 2012. The impact of the contributor in VGI projects, in: Workshop on Map Creation from User Generated Data, Hannover, Germany, 8 October 2012. Available at: http://www. visualisierung.dgfk.net/docs/Schmidt-etal.pdf [Last accessed 16 May 2017].

Sedano, E., 2016. “Sensor"ship and Spatial Data Quality. Urban Planning, 1(2), 75-87. DOI: https://doi.org/10.17645/up.v1i2.608

See, L., Mooney, P., Foody, G., Bastin, L., Comber, A., Estima, J., Fritz, S., Kerle, N., Jiang, B., Laakso, M., Liu, H.-Y., Milčinski, G., Nikšič, M., Painho, M., Pődör, A., Olteanu-Raimond, A.-M., Rutzinger, M., 2016. Crowdsourcing, citizen science or Volunteered Geographic Information? The current state of crowdsourced geographic information. ISPRS International Journal of Geo-Information 5, 55. DOI: https://doi.org/10.3390/ijgi5050055

Skopeliti, A, Antoniou, V and Bandrova, T. 2017. Visualisation and Communication of VGI Quality. In: Foody, G, See, L, Fritz, S, Mooney, P, Olteanu-Raimond, A-M, Fonte, C C and Antoniou, V. (eds.) Mapping and the Citizen Sensor. Pp. 197-222. London: Ubiquity Press. DOI: https://doi. org/10.5334/bbf.i.

Turner, A., 2006. Introduction to Neogeography. O’Reilly, Sebastopol, Calif.

van Exel, M., Dias, E., Fruijtier, S., 2010. The impact of crowdsourcing on spatial data quality indicators, in: Wallgrün, J.O., Lautenschütz, A.K. (eds.), Proceedings of the GIScience 2010 Doctoral Colloquium, Zurich, Switzerland, 14-17 September 2010. Available at: http://www.giscience2010.org/ pdfs/paper_213.pdf [Last accessed 16 May 2017]

Viglino, J.-M., 2009. Managing partners feedbacks through the geoweb, in: Streilein, A. \& Kellenberger, T. (eds.), 2010. Proceedings of the EuroSDR Workshop 'Crowd Sourcing for Updating National Databases' held from 20th to 21st August 2009 in Wabern, Switzerland.

Yanenko, O., Schlieder, C., 2014. Game principles for enhancing the quality of user-generated data collections. Presented at the AGILE 2014 Workshop on Geogames, Castellón, Spain, pp. 1-5. 